\title{
The relationship between sarcopenia and nesfatin- 1 and ghrelin levels in patients with chronic obstructive pulmonary disease
}

\author{
๑Gülay Daşdemir İlkhan¹, @Selay Arslan², ๑Yücel Arman³, ®Özgür Altun³, @Hakan Çelikhisar, \\ (D)Tufan Tükek ${ }^{5}$ \\ ${ }^{1}$ Tire State Hospital, Department of Chest Diseases, İzmir, Turkey \\ ${ }^{2}$ Okmeydanı Training and Research Hospital, Department of Chest Diseases, İstanbul, Turkey \\ ${ }^{3}$ Okmeydanı Training and Research Hospital, Department of Internal Medicine, İstanbul, Turkey \\ ${ }^{4}$ İzmir Metropolitan Municipality Hospital, Department of Chest Diseases, İzmir, Turkey \\ ${ }^{5}$ İstanbul University, İstanbul Faculty of Medicine, Department of Internal Medicine, İstanbul, Turkey
}

Cite this article as: Daşdemir İlkhan G, Arslan S, Arman Y, Altun Ö, Çelikhisar H, Tükek. The relationship between sarcopenia and nesfatin-1 and ghrelin levels in patients with chronic obstructive pulmonary disease. J Health Sci Med 2021; 4(4): 402-407.

\begin{abstract}
Aim: In this study, we aimed to investigate the relationship between the levels of adipocytokines anorexigenic nesfatin-1 and orexigenic ghrelin levels and body mass index (BMI), muscle mass, muscle strength and sarcopenia in patients with chronic obstructive pulmonary disease (COPD).

Material and Method: The patients were divided into two groups according to the severity of COPD. Body mass index (BMI), body fat and muscle measurements were measured with bioelectrical impedance device. A hand-grip strength test was performed with a hand dynamometer to determine muscle strength. We used the mMRC (Modified Medical Research Council Questionnaire) questionnaire to measure the intensity of dyspnea during daily activities. A 6-minute walk test (6MWT) was used to test functional capacity and physical exercise tolerance. COPD Assessment Test (CAT) was performed to determine the health status. Serum nesfatin-1 and ghrelin levels were measured in all patients.

Results: In a total of 90 patients (16 female and 74 male) were included in the study prospectively. There was not any significant difference regarding the Nesfatin-1, ghrelin and Nesfatin-1/ghrelin ratio between mild/moderate or severe COPD patients. Muscle strength was correlated positively with 6MWT, nesfatin-1 and ghrelin levels, but negatively correlated with CAT score and $\mathrm{mMRC}$ score. The number of patients in the advanced stage of COPD was higher in the sarcopenia group than in the non-sarcopenia group.

Conclusion: There was a positive correlation between muscle strength and nesfatin-1 and ghrelin levels. However, muscle strength was negatively correlated with mMRC and CAT scores. Approximately 19\% of COPD patients had sarcopenia. Serum nesfatin-1 and ghrelin levels were not different between sarcopenic and non-sarcopenic COPD patients.
\end{abstract}

Keywords: Chronic obstructive pulmonary disease, ghrelin, nesfatin-1, sarcopenia

\section{INTRODUCTION}

Chronic obstructive pulmonary disease (COPD) is characterized by permanent limitation of airflow and exacerbations in respiratory distress, also causing systemic effects. In addition to the inflammation process in the lungs, there is a low-grade systemic inflammation in COPD resulting in co-morbidities (1). Although not elucidated clearly yet; smoking, lung hyperinflation, tissue hypoxia and skeletal muscle dysfunction have been suggested as possible factors in the pathogenesis of systemic inflammation in COPD. Lately, adipose tissue mediated inflammation is gaining increasing interest as an important mechanism in inducing systemic inflammation in COPD (2).

Involuntary weight loss is a common complication of COPD and has been found to be associated with negative outcomes regardless of its degree. It was found that approximately $50 \%$ of hospitalized patients with COPD were below $90 \%$ of their ideal body weight. Low body weight and muscle loss are associated with accelerated 
mortality and decline in clinical status, regardless of the severity of lung disease. Sarcopenia is defined as low muscle mass together with low muscle function. Initially, sarcopenia was used to describe age-related muscle mass and loss of strength. The European Working Group on Sarcopenia in the Elderly (EWGSOP) recommends the presence of both low muscle mass and low muscle function (strength or performance) for the diagnosis of sarcopenia (3). Chronic inflammatory diseases are known to induce sarcopenia $(4,5)$. Recently, in presarcopenic and sarcopenic individuals a higher prevalence of COPD but not other chronic diseases was reported (6).

Adipokines are protein mediators secreted by adipose tissue and are involved not only in regulating energy metabolism, but also in inflammatory responses in many chronic diseases. Nesfatin-1 is a new adipokine that was discovered in 2006 and was first associated with appetite and body weight control in rats. Nesfatin-1 is expressed in human adipose tissue and has been shown to increase secretion of tumor necrosing factor- $\alpha$ (TNF- $\alpha$ ), IL6, insulin and dexamethasone (7). On the other hand, a study examining emphysema-type COPD patients suggested that NUCB2/nesfatin-1 may be a new factor associated with systemic inflammation in COPD and may emerge as a stable new inflammatory factor (8). Ghrelin, a 28 -amino acid peptide secreted from the stomach, was isolated in 1999 as an endogenous ligand for the growth hormone secretor receptor. Plasma ghrelin levels are inversely proportional to body mass index (9).

In this study, we aimed to investigate the relationship between circulating nesfatin-1 and ghrelin levels and body mass index, muscle mass, muscle strength and sarcopenia in COPD patients; this may lead to a new way of predicting the course of the disease and delaying and reducing complications.

\section{MATERIAL AND METHOD}

In this prospective study, the patients with mild, moderate, severe and very severe stable COPD who were admitted to the pulmonology outpatient clinic during the three-month period (between 30 June 2019 and 30 September 2019) were included in the study. Patients with malignancy, thyroid dysfunction, psychiatric disorders or chronic inflammatory diseases, and patients receiving corticosteroid therapy or dietary supplementation were excluded. All consecutive patients who gave consent were included in the study. The study was approved by the Health Sciences University, Okmeydanı Training and Research Hospital Clinical Research Ethics Committee (Date: 23.06.2019, Decision No: 48670771-514.10) and performed in accordance with declaration of Helsinki.
In addition to demographic characteristics, body mass index (BMI), body fat and muscle measurements were recorded with bioelectrical impedance device (Tanita BIA model TBF-300; Tanita Corporation, Tokyo, Japan). To determine the muscle strength, a handgrip strength test was performed with a hand dynamometer (Jamar dynamometer, Takei Scientific Instruments Co., LtdJapan). While the grip strength was measured, three measurements were performed with the dominant hand and averaged in kilograms (3). BMI was calculated as body weight $(\mathrm{kg}) /$ height $2(\mathrm{~m} 2)$. The cut-off values for low muscle mass were $\leq 8.50 \mathrm{~kg} / \mathrm{m} 2$ for men and $\leq 5.75$ $\mathrm{kg} / \mathrm{m} 2$ for women (3). To evaluate the muscle strength, grip strength was considered as $>20 \mathrm{~kg}$ in women and $>$ $30 \mathrm{~kg}$ in men as normal (3). According to the European Working Group on Sarcopenia in the Elderly, patients with low muscle mass and low muscle strength were evaluated as having sarcopenia (3).

According to GOLD criteria, the post-bronchodilator FEV1/FVC $<70 \%$ on spirometric measurement confirms the diagnosis of COPD. Patients were classified as $\geq 80 \%$ (mild), $50-79 \%$ (moderate), $30-49 \%$ (severe) and $<30 \%$ (very severe) according to the expected FEV1 percentage on spirometric measurement (10). At the same time, the severity of COPD (clinical stage) was determined according to the GOLD criteria, with the postbronchodilator FEV 1, the number of exacerbations on the last year, presence of hospitalization, and dyspnea. Dyspnea was evaluated with the mMRC and CAT scores (10). Based on these criteria, each patient was clinically classified as GOLD stage A, B, C or D (10). We divided the patients into two groups for comparison. Patients with COPD were classified as a group $(1+2)$ with mild to moderate obstructive disorder according to FEV1 values, and the remaining group with severe to very severe obstructive disorder $(3+4)$. COPD was also divided into two groups according to the clinical stage $\mathrm{A}+\mathrm{B}$ and $\mathrm{C}+\mathrm{D}$. We used the mMRC questionnaire to measure the intensity of dyspnea during daily activities. COPD Assessment Test was performed to determine the health status. Patients with a total score of $\geq 10$ points were evaluated as symptomatic (11). 6MWT was used to test the functional capacity and physical exercise tolerance.

Serum nesfatin-1 and ghrelin levels were measured in all patients. For blood Nesfatin-1 and ghrelin measurements, blood was transferred to yellow biochemistry tube, centrifugation was carried out, and supernatant was taken to eppendorf tube and stored at -80 degrees. Serum levels of Nesfatin-1 and ghrelin were detected by ELISA (enzyme-linked immunosorbent assay) method through commercial kits. 


\section{Statistical Analyses}

Statistical analyses were performed by using SPSS program v.21 (SPSS Inc., Chicago, IL). Continuous data were expressed as mean \pm standard deviation or median and categorical data were expressed as percentages. Chisquare test was used for the comparison of categorical data. Pearson's correlation analysis was performed to determine the association of different test parameters. A value of $p<0.05$ was considered statistically significant.

\section{RESULTS}

In a total of 90 patients ( 16 female and 74 male) were included in this prospective study. The mean age of the study participants was $59.82 \pm 9.984$ years. Patients with COPD were divided into subgroups according to the spirometric FEV1 (\%) measurements as mild-moderate (stage 1 and 2) and severe-very severe (stage 3 and 4). Between those two subgroups, BMI, fat mass, muscle mass and grip strength, serum nesfatin-1, ghrelin and nesfatin-1/ghrelin ratio were compared (Table 1). In the mild to moderate COPD group; the number of patients with normal fat mass (\%) was significantly higher. There was no significant difference between the two subgroups of COPD patients in terms of nesfatin-1, ghrelin levels or nesfatin-1/ghrelin ratio.

\begin{tabular}{|c|c|c|c|}
\hline & $\begin{array}{c}\text { GOLD 1+2 } \\
(\mathrm{n}: 49)\end{array}$ & $\begin{array}{c}\text { GOLD 3+4 } \\
(\mathrm{n}: 41)\end{array}$ & $P$ value \\
\hline BMI (kg/m2) & $25.25 \pm 4.58$ & $23.47 \pm 4.31$ & 0.09 \\
\hline Fat mass (\%) & $24.07 \pm 10.37$ & $19.33 \pm 9.17$ & 0.026 \\
\hline Muscle mass (\%) & $33.03 \pm 4.99$ & $33.65 \pm 4.40$ & 0.541 \\
\hline Grip strength (kg) & $32.03 \pm 7.12$ & $29.66 \pm 6.74$ & 0.113 \\
\hline $6 \mathrm{MWT}(\mathrm{m} / \mathrm{sn})$ & $0.92 \pm 0.08$ & $0.77 \pm 0.14$ & 0.001 \\
\hline CAT score $(+)$ & $3 / 49$ & $29 / 41$ & 0.001 \\
\hline mMRC score & $1.29 \pm 0.50$ & $2.74 \pm 0.71$ & 0.001 \\
\hline Nesfatin-1(ng/ml) & $19.64 \pm 15.71$ & $22.45 \pm 14.68$ & 0.385 \\
\hline Ghrelin (ng/ml) & $2.74 \pm 3.83$ & $2.92 \pm 3.02$ & 0.803 \\
\hline Nesfatin-1/Ghrelin ratio & $8.62 \pm 3.48$ & $9.10 \pm 3.09$ & 0.493 \\
\hline
\end{tabular}

Patients were subdivided into mild-moderate (stage A and $\mathrm{B}$ ) and severe-very severe (stage $\mathrm{C}$ and $\mathrm{D}$ ) groups according to the GOLD clinical stage. BMI, fat mass, muscle mass and grip strength, serum nesfatin-1, ghrelin and nesfatin-1/ghrelin ratio between these two subgroups were compared (Table 2). The number of patients with normal fat mass (\%) and 6MWT was significantly higher in the mild to moderate COPD group. There was no significant difference between the two subgroups of COPD patients in terms of nesfatin-1, ghrelin and nesfatin-1/ghrelin ratio.
Table 2. Comparison of BMI, fat mass and grip strength, serum nesfatin-1, ghrelin and nesfatin-1/ghrelin ratio

\begin{tabular}{|lccc|}
\hline & $\begin{array}{c}\text { GOLD (A+B) } \\
(\mathbf{n}: 47)\end{array}$ & $\begin{array}{c}\text { GOLD (C+D) } \\
\text { (n:43) }\end{array}$ & $\begin{array}{c}\text { P } \\
\text { value }\end{array}$ \\
\hline BMI (kg/m2) & $24.80 \pm 4.76$ & $24.12 \pm 4.27$ & 0.47 \\
Fat mass (\%) & $23.02 \pm 11.00$ & $20.92 \pm 9.01$ & 0.032 \\
Muscle mass (\%) & $33.22 \pm 5.37$ & $33.39 \pm 3.97$ & 0.86 \\
Grip strength (kg) & $32.00 \pm 7.63$ & $29.93 \pm 6.20$ & 0.16 \\
6MWT(m/sn) & $0.92 \pm 0.08$ & $0.78 \pm 0.14$ & 0.001 \\
CAT score (+) & $7 / 47$ & $25 / 43$ & 0.001 \\
mMRC score & $1.51 \pm 0.65$ & $2.37 \pm 1.00$ & 0.001 \\
Nesfatin-1(ng/ml) & $20.25 \pm 15.74$ & $21.52 \pm 14.85$ & 0.69 \\
Ghrelin (ng/ml) & $2.84 \pm 3.97$ & $2.79 \pm 2.90$ & 0.94 \\
Nesfatin-1/Ghrelin ratio & $8.84 \pm 3.68$ & $8.82 \pm 2.90$ & 0.98 \\
\hline
\end{tabular}

BMI:Body mass index, 6MWT:6 minutes walking test, CAT: COPD assessment test, mMRC: Modified Medical Research Council

In correlation analysis, fat mass was positively correlated with BMI and negatively correlated with mMRC score; muscle mass was negatively correlated with BMI. Grip strength was positively correlated with 6MWT, nesfatin-1 and ghrelin levels, but negatively correlated with CAT and mMRC scores (Table 3).

Table 3. Correlation analysis between patients' clinical characteristics and fat mass, muscle mass and handgrip strength

\begin{tabular}{|c|c|c|c|c|c|c|}
\hline & \multicolumn{2}{|c|}{ Fat mass (\%) } & \multicolumn{2}{|c|}{$\begin{array}{c}\text { Muscle mass } \\
(\%)\end{array}$} & \multicolumn{2}{|c|}{ Grip strength } \\
\hline & $\mathbf{r}$ & $\mathbf{p}$ & $\mathbf{r}$ & $\mathbf{p}$ & $\mathbf{r}$ & $\mathbf{p}$ \\
\hline Age (years) & 0.024 & 0.826 & -0.148 & 0.165 & -0.123 & 0.248 \\
\hline $\mathrm{BMI}(\mathrm{kg} / \mathrm{m} 2)$ & 0.678 & 0.001 & -0.469 & 0.001 & 0.077 & 0.468 \\
\hline 6MWT (m/sn) & 0.101 & 0.343 & 0.124 & 0.245 & 0.237 & 0.024 \\
\hline CAT score $(+)$ & -0.172 & 0.105 & -0.001 & 0.994 & -0.244 & 0.021 \\
\hline mMRC score & -0.243 & 0.021 & 0.013 & 0.900 & -0.305 & 0.003 \\
\hline $\begin{array}{l}\text { Nesfatin-1 } \\
\text { (ng/ml) }\end{array}$ & -0.158 & 0.137 & 0.170 & 0.108 & 0.221 & 0.036 \\
\hline $\begin{array}{l}\text { Ghrelin } \\
\text { (ng/ml) }\end{array}$ & -0.152 & 0.152 & 0.190 & 0.073 & 0.241 & 0.022 \\
\hline $\begin{array}{l}\text { Nesfatin-1/ } \\
\text { Ghrelin ratio }\end{array}$ & -0.003 & 0.978 & -0.017 & 0.873 & -0.055 & 0.607 \\
\hline
\end{tabular}

Sarcopenia was detected in 17 of the study participants. A comparison of the general characteristics and laboratory results of patients with or without sarcopenia is summarized in Table 4. There was no significant difference between the two groups in terms of age, gender, BMI or smoking status. However, the total cigarette pack/year (p: 0.04) and COPD duration (p: 0.02 ) of the patients with sarcopenia were significantly higher than those of COPD patients without sarcopenia. Similarly, the number of patients in the advanced stage of COPD was higher in the sarcopenia group than in the non-sarcopenia group. Also, FEV1 (\%), FVC (\%) and FEV1/FVC (\%) values were worse in sarcopenia group compared to non-sarcopenia group (p: 0.001). 


\begin{tabular}{|c|c|c|c|}
\hline & $\begin{array}{c}\text { Sarcopenia } \\
(+)(\mathrm{n}: 17)\end{array}$ & $\begin{array}{c}\text { Sarcopenia } \\
(-)(\mathbf{n}: 73)\end{array}$ & $\begin{array}{c}\mathrm{P} \\
\text { value }\end{array}$ \\
\hline Age (years) & $62.00 \pm 6.432$ & $58.90 \pm 9.872$ & 0.22 \\
\hline Gender (F/M) & $0 / 17$ & $16 / 57$ & 0.035 \\
\hline Smoking (quitted /still smoking) & $7 / 10$ & $24 / 49$ & 0.68 \\
\hline Cigarette (pocket x years) & $43.11 \pm 11.52$ & $36.16 \pm 15.49$ & 0.04 \\
\hline BMI $(\mathrm{kg} / \mathrm{m} 2)$ & $23.62 \pm 4.51$ & $24.68 \pm 4.53$ & 0.39 \\
\hline $\begin{array}{l}\text { GOLD stage (Predicted FEV1\%) } \\
1+2 \\
3+4\end{array}$ & $\begin{array}{c}3 \\
14\end{array}$ & $\begin{array}{l}48 \\
25\end{array}$ & 0.001 \\
\hline $\begin{array}{l}\text { GOLD stage (Clinical) } \\
A+B \\
C+D\end{array}$ & $\begin{array}{c}2 \\
15\end{array}$ & $\begin{array}{l}45 \\
28\end{array}$ & 0.001 \\
\hline COPD duration (years) & $8.76 \pm 6.13$ & $4.87 \pm 4.49$ & 0.02 \\
\hline FEV1 $(\%)$ & $44.58 \pm 19.40$ & $66.10 \pm 19.31$ & 0.001 \\
\hline FVC (\%) & $58.29 \pm 15.75$ & $74.16 \pm 18.58$ & 0.001 \\
\hline FEV1/FVC\% & $58.64 \pm 13.52$ & $69.17 \pm 8.31$ & 0.001 \\
\hline CAT score $(+) /(-)$ & $14 / 3$ & $18 / 55$ & 0.001 \\
\hline 6MWT (m/sn) & $0.62 \pm 0.06$ & $0.91 \pm 0.07$ & 0.001 \\
\hline mMRC score & $2.76 \pm 1.09$ & $1.72 \pm 0.78$ & 0.001 \\
\hline CRP & $5.31 \pm 1.33$ & $4.83 \pm 1.75$ & 0.29 \\
\hline \multicolumn{4}{|c|}{$\begin{array}{l}\text { BMI: Body Mass Index, GOLD: Global Strategy for the Diagnosis, Management and } \\
\text { Prevention of Chronic Obstructive Pulmonary Disease, FEV1: Forced expiratory volume } \\
\text { on the first second, FVC: Forced vital capacity, CAT: COPD assessment test, } 6 \text { MWT: } 6 \\
\text { Minute Walking test, mMRC: Modified Medical Research Council }\end{array}$} \\
\hline
\end{tabular}

Fat mass, muscle mass and grip strength were compared between the sarcopenia and non-sarcopenia groups, and as expected, the muscle mass and grip strength were worse in the sarcopenia group (p: 0.001) (Table 5).

\begin{tabular}{|c|c|c|c|}
\hline & $\begin{array}{c}\text { Sarcopenia } \\
(+)(\mathrm{n}: 17)\end{array}$ & $\begin{array}{c}\text { Sarcopenia (-) } \\
(\mathrm{n}: 73)\end{array}$ & $\begin{array}{c}P \\
\text { value }\end{array}$ \\
\hline Fat mass (\%) & $21.48 \pm 7.84$ & $22.14 \pm 10.60$ & 0.77 \\
\hline Muscle mass (\%) & $31.44 \pm 1.44$ & $33.73 \pm 5.11$ & 0.001 \\
\hline Grip strength (kg) & $28.52 \pm 3.44$ & $31.58 \pm 7.52$ & 0.001 \\
\hline Nesfatin-1 (ng/ml) & $25.46 \pm 16.97$ & $19.789 \pm 14.74$ & 0.21 \\
\hline Ghrelin (ng/ml) & $3.15 \pm 2.43$ & $2.74 \pm 2.52$ & 0.66 \\
\hline Nesfatin-1/Ghrelin ratio & $9.68 \pm 3.44$ & $8.63 \pm 3.27$ & 0.26 \\
\hline
\end{tabular}

\section{DISCUSSION}

In this study, we analyzed the association of circulating nesfatin-1 and ghrelin levels with muscle loss, muscle strength, and sarcopenia in COPD patients and we determined that; 1) Fat mass (\%) was significantly lower in patients with severe COPD than patients with mild to moderate COPD. Although the muscle mass did not change significantly in the severe COPD group, physical performance was significantly lower with low fat mass; 2 ) In correlation analysis, fat mass was positively correlated with BMI and negatively correlated with mMRC score; muscle mass correlated negatively with BMI; handgrip strength was positively correlated with 6MWT, nesfatin-1 and ghrelin levels, but negatively correlated with CAT and mMRC scores; 3) Total cigarette pack/year smoking and
COPD duration were significantly higher in sarcopenia patients; FEV1 (\%), FVC (\%) and FEV1/FVC (\%), CAT score, $6 \mathrm{MWT}$ and $\mathrm{mMRC}$ score were worse than patients without sarcopenia; 4) When patients were compared for the presence of sarcopenia or COPD, the ratio of nesfatin-1, ghrelin and nesfatin-1/ghrelin did not differ significantly.

When the mild and severe patient groups with COPD were compared, fat mass was decreased, muscle mass was preserved and BMI was not changed in severe COPD patients. Our patients are outpatients, and their mobility may explain the relative protection of muscle masses while decreasing fat masses. In a study evaluating nesfatin-1 levels in patients with cystic fibrosis (12), high nesfatin-1 levels with low fat mass were detected in patients with severe cystic fibrosis. In our study, there was no significant change in nesfatin-1 values with decreased fat mass in severe COPD patients. Circulating ghrelin levels are generally positively proportional to weight loss and fat mass reduction (9), but we did not observe a change in ghrelin levels in patients with severe COPD, with a decrease in fat mass. While the effect of adipokines nesfatin-1 and ghrelin on fat tissue was expected, no significant changes were observed in nesfatin-1 and ghrelin levels, although fat mass decreased in severe COPD patients. With the introduction of different mechanisms in COPD, the process may be altered differently in changes in body composition.

Studies on the relationship between fat mass and COPD are limited. Both being overweight or obese, have been shown to increase the severity of symptoms in patients with COPD (13). However, in a recent study, in contrast to previous findings, the mortality of overweight and obese patients with COPD was shown to be low (14). Existing studies have been performed mainly with overweight and obese COPD patients. In general, there are only a small number of studies investigating patients with COPD having normal or lower BMI. In a retrospective cohort study (15) investigating the exacerbation risk of COPD patients with a predominantly normal and low BMI, fat mass showed a significant protective effect for acute exacerbation of COPD. In our study, fat mass was decreased, muscle mass was preserved in patients with severe COPD with similar BMI, but a decrease in physical performance was observed. In spite of the possible negative effects of fat mass, especially in obese COPD patients, the protective effect of fat mass in COPD patients with normal BMI was quite significant.

In the study of Yamamoto et al (16), it was found that TNF- $\alpha$ (tumor necrosis factor-alpha) levels increased in stable COPD cases and there was a negative correlation between fat mass and TNF- $\alpha$. Fat mass may play a 
protective role in COPD. TNF- $\alpha$, a good indicator of inflammation, is inversely proportional to fat mass. In our study, this finding supports the decrease in physical performance in patients with severe COPD with decreasing fat tissue and increasing the severity of inflammation.

There is significant interest in understanding the mechanisms underlying low muscle mass in patients with COPD. Systemic inflammation, oxidative stress, hypoxia, and hypercapnia are the main mechanisms suggested in sarcopenia (2). Poberezhets et al (17) examined 98 male COPD patients with a mean age of $60.4 \pm 11.2$ years and reported that, increase in the frequency of COPD exacerbations was associated with the decrease in the 6-minute walk test distance and with the rise of sarcopenia. Graumam et al (18) reported that, with an increase in disease severity, there was a decrease in skeletal mass index and increase in the rate of low lean mass in COPD patients. In a cross-sectional study of 240,562 Korean adults without known lung disease, decreased skeletal muscle mass index was determined to be independently associated with a decrease in lung function in actually healthy adults (19). In this study, we also determined worse FEV1, FVC and FEV1/FVC results in patients with sarcopenia. Moreover, with an increase in COPD severity, fat mass was decreasing and the number of patients with low muscle mass was increasing.

Another important finding of this study was the correlation of handgrip strength with 6MWT, Nesfatin-1 and Ghrelin levels, and inverse correlation with CAT score positivity and mMRC score. Similar with our results Kyomoto et al (20) reported that carbon monoxide diffusing capacity of the lungs and handgrip strength were strongly correlated with 6MWT in their study on 133 patients with COPD. In previous literature, decreased ghrelin levels were associated with the nutritional status of the COPD patients and ghrelin has been suggested for the treatment of COPD patients (21-23). However, we did not determine any significant difference regarding ghrelin levels between COPD patients with or without sarcopenia or any alteration in ghrelin levels with an advance in COPD.

In previous literature, the data analyzing the association of COPD with sarcopenia is limited. The prevalence of sarcopenia in patients with COPD was reported between $15 \%$ and $25 \%$ (24). In 622 outpatients with stable COPD, Jones et al reported the Prevalence of sarcopenia as $14.5 \%$ (25). The prevalence of cachexia, which was defined as unintentional weight loss $>5 \%$ and low fatfree mass index, was reported as $4.6 \%$ in 1755 outpatients with stable COPD (26). In our study the prevalence of sarcopenia was $18.8 \%$, which was compatible with the literature.
Another finding of the study was that in patients with sarcopenia, CAT score, 6MWT and mMRC score were all worse compared with the patients without sarcopenia. Pothirat et al (27) reported a significant correlation between fat-free mass index and $\mathrm{mMRC}, \mathrm{FEV}_{1}$, and CAT score in 121 patients with stable COPD. In that study, the rate of sarcopenia/ muscular atrophy was 9.9, and the rate of cachexia was as high as $35.5 \%$.Similar with our results, Gologanu et al (28) reported that the sarcopenic COPD patients were having the lowest mean 6MWT and the higher CAT mean scores. In a crosssectional study, Serra pratt et al (29) investigated the association of plasma ghrelin levels and sarcopenia in elderly people and reported significantly lower ghrelin levels in elderly patients with sarcopenia but this difference disappeared when stratified for gender. We did not determine any significant difference regarding ghrelin or nesfatin-1 levels between sarcopenic and non-sarcopenic COPD patients or with an advance in disease severity. In a recent meta-analysis, circulating level of ghrelin was reported to be significantly elevated in patients with COPD, especially in those underweight (30).

There are some limitations that should be mentioned. First is the low number of patients and secondly, followup data was not analyzed in this study.

\section{CONCLUSION}

In conclusion, while the effect of nesfatin- 1 and ghrelin on fat mass was expected in COPD patients, there was no significant change in nesfatin-1 and ghrelin levels in patients with severe COPD although there was a decrease in fat mass. The decrease in fat mass in severe COPD, decreased physical performance even though muscle mass did not change. Fat mass in patients with severe COPD with normal and low BMI, may play a protective role in the course of the disease. Muscle strength was positively correlated with nesfatin-1 and ghrelin levels, but negatively correlated with mMRC and CAT scores. Approximately 19\% of COPD patients had sarcopenia. When the patients were compared for the presence of sarcopenia or COPD; nesfatin-1, ghrelin and nesfatin-1/ ghrelin ratio did not differ significantly. Larger studies with longer follow-up periods are needed to determine the exact role of nesfatin-1 or ghrelin levels in COPD patients and to guide their role in treatment..

\section{ETHICAL DECLARATIONS}

Ethics Committee Approval: The study was approved by the Health Sciences University, Okmeydanı Training and Research Hospital Clinical Research Ethics Committee (Date: 23.06.2019, Decision No: 48670771-514.10). 
Informed Consent: All patients signed the free and informed consent form.

Referee Evaluation Process: Externally peer-reviewed.

Conflict of Interest Statement: The author(s) declared no potential conflicts of interest with respect to the research, authorship, and/or publication of this article.

Financial Disclosure: The authors have not declared a specific grant for this research from any funding agency in the public, commercial or not-for-profit sectors.

Author Contributions: All of the authors declare that they have all participated in the design, execution, and analysis of the paper, and that they have approved the final version.

\section{REFERENCES}

1. Barnes PJ, Celli BR. Systemic manifestations and comorbidities of COPD. Eur Respiratory J 2009; 33: 1165-85.

2. Maltais F, Decramer M, Casaburi R, et al. An official American Thoracic Society/European Respiratory Society statement: update on limb muscle dysfunction in chronic obstructive pulmonary disease. Am J Respir Crit Care Med 2014; 189: e15-e62.

3. Cruz-Jentoft AJ, Baeyens JP, Bauer JM, et al. Sarcopenia European consensus on definition and diagnosis: report of the European Working Group on Sarcopenia in Older People. Age Ageing 2010; 39: 412-23.

4. Bauer J, Morley JE, Schols AMWJ, et al. Sarcopenia: a time for action. An SCWD Position Paper. J Cachexia Sarcopenia Muscle 2019; 10: 956-61.

5. Dziegala M, Josiak K, Kasztura M, et al. Iron deficiency as energetic insult to skeletal muscle in chronic diseases. J Cachexia Sarcopenia Muscle 2018; 9: 802-15.

6. Trajanoska K, Schoufour JD, Darweesh SK, et al. Sarcopenia and its clinical correlates in the general population: The Rotterdam Study. J Bone Miner Res 2018; 33: 1209-18.

7. Algül S, Özçelik O. Determination of the effects of acute aerobic exercise on nesfatin-1 levels. Firat University Med J Health Sci 2016; 30: 5-8.

8. Leivo-Korpela $S$, Lehtimäki L, Hämälainen $M$, et al. Adipokines NUCB2/nesfatin-1 and visfatin as novel inflammatory factors in chronic obstructive pulmonary disease. Mediators Inflamm 2014; 2014: 232167.

9. Gil-Campos M, Aguilera CM, Cañete R, Gil A. Ghrelin: a hormone regulating food intake and energy homeostasis. Br J Nutr 2006; 96: 201-26.

10. Disease. GIfCOL. Global Strategy for the Diagnosis, Management and Prevention of COPD. Global initiative for chronic obstructive lung diseases (GOLD). 2014. Available from: http://www. goldcopd.org. Accessed January 1.

11. Jones PW, Harding G, Berry P, Wiklund I, Chen WH, Kline Leidy N. Development and first validation of the COPD Assessment Test. Eur Respir J 2009; 34: 648-54.

12. Cohen RI, Ginsberg N, Tsang D, Wann LC, Ye X, Liu SF. Association of nesfatin-1 and fat mass in cystic fibrosis. Respiration 2013; 86: 312-7.

13.Zammit C, Liddicoat H, Moonsie I, Makkeer H. Obesity and respiratory diseases. Int J Gen Med 2010; 3: 335-43.

14. Spelta F, Fratta Pssini AM, Cazzoletti L, Ferrari M. Body weight and mortality in COPD: focus on the obesity paradox. Eat Weight Disord 2018; 23: 15-22.
15. Yang L, Zhu Y, Huang JA, Jin J, Zhang X. A low lean-to-fat ratio reduces the risk of acute exacerbation of chronic obstructive pulmonary disease in patients with a normal or low body mass index. Med Sci Monit 2019; 25: 5229-36.

16. Yamamoto C, Yoneda T, Yoshikawa M, et al. The relationship between a decrease in fat mass and serum levels of TNF-alpha in patients with chronic obstructive pulmonary disease. Nihon Kyobu Shikkan Gakkai Zasshi 1997; 35: 1191-5.

17. Poberezhets V, Mostovoy Y, Demchuk H. Exacerbation of chronic obstructive pulmonary diseases as a risk factor of the skeletal muscle dysfunction. Lung India 2019; 36: 188-92.

18. Graumam RQ, Pinheiro MM, Nery LE, Castro CHM. Increased rate of osteoporosis, low lean mass, and fragility fractures in COPD patients: association with disease severity. Osteoporos Int 2018; 29: 1457-68.

19. Park CH, Yi Y, Do JG, Lee YT, Yoon KJ. Relationship between skeletal muscle mass and lung function in Korean adults without clinically apparent lung disease. Medicine (Baltimore) 2018; 97: e12281.

20. Kyomoto Y, Asai K, Yamada K, et al. Handgrip strength measurement in patients with chronic obstructive pulmonary disease: Possible predictor of exercise capacity. Respir Investig 2019; 57: 499-505.

21.Itoh T, Nagaya N, Yoshikawa M, et al. Elevated plasma ghrelin level in underweight patients with chronic obstructive pulmonary disease. Am J Respir Crit Care Med 2004; 170: 879-82.

22.Deveci Y, Deveci F, Ilhan N, Karaca I, Turgut T, Muz MH. Serum ghrelin, IL-6 and TNF- $\alpha$ levels in patients with chronic obstructive pulmonary disease. Tuberk Toraks 2010; 58: 162-7. Turkish.

23. Kamiide Y, Inomata N, Furuya M, Yada T. Ghrelin ameliorates catabolic conditions and respiratory dysfunction in a chronic obstructive pulmonary disease model of chronic cigarette smokeexposed rats. Eur J Pharmacol 2015; 755: 88-94.

24. Kim SH, Shin MJ, Shin YB, Kim KU. Sarcopenia Associated with Chronic Obstructive Pulmonary Disease. J Bone Meta 2019; 26: 65-74.

25. Jones SE, Maddocks M, Kon SS, et al. Sarcopenia in COPD: prevalence, clinical correlates and response to pulmonary rehabilitation. Thorax 2015; 70: 213-8.

26. Kwan HY, Maddocks M, Nolan CM, et al. The prognostic significance of weight loss in chronic obstructive pulmonary disease-related cachexia: a prospective cohort study. J Cachexia Sarcopenia Muscle 2019; 10: 1330-8.

27. Pothirat C, Chaiwong W, Phetsuk N, et al. The Relationship between Body Composition and Clinical Parameters in Chronic Obstructive Pulmonary Disease.J Med Assoc Thai 2016; 99: 38693.

28. Gologanu D, Ionita D, Gartonea T, Stanescu C, Bogdan MA. Body composition in patients with chronic obstructive pulmonary disease. Maedica (Buchar) 2014; 9: 25-32.

29.Serra-Prat M, Papiol M, Monteis R, Palomera E, Cabré M. Relationship between Plasma Ghrelin Levels and Sarcopenia in Elderly Subjects: A Cross-Sectional Study. J Nutr Health Aging 2015; 19: 669-72.

30.Zhang X, Yang T, Wang J, et al. Elevated circulating ghrelin in patients with COPD: A meta-analysis.Chron Respir Dis 2018; 15: 365-73. 